\title{
Metabolomics reveals changes in urea cycle associated to residual feed intake in growing heifers
}

Jorge-Smeding, E. ${ }^{1}$, G. Renand ${ }^{2}$, D. Centeno ${ }^{3}$, M. Pétéra ${ }^{3}$, S. Durand ${ }^{3}$, S. Polakof ${ }^{4}$, G. Cantalapiedra-Hijar ${ }^{1}$

${ }^{1}$ INRA, UMR Herbivores, F63122 Saint-Genès-Champanelle, France.

${ }^{2}$ INRA, UMR Génétique Animale et Biologie Intégrative, F78352 Jouy-en-Josas, France

${ }^{3}$ INRA, UMR PFEM, UNH, F63000 Clermont-Ferrand, France

${ }^{4}$ INRA, UMR Unité de Nutrition Humaine, F-63000 Clermont-Ferrand, France.

\begin{abstract}
Previous studies exploring biomarkers of residual feed intake (RFI) in beef cattle have been conducted on fast-growing males. However, predicting RFI in females is also important because the cowherd represents most of the feeding cost of the overall system. In order to identify candidate biomarkers of RFI in growing females, 48 Charolais heifers with the highest and lowest RFI were selected from 142 individuals to study their blood plasma metabolome through a non-targeted metabolomics approach. The analysis highlighted 147 ions potentially of interest regarding the RFI classes (raw p-values from ANOVA: $P<0.1$, of which $n=74$ with $P<0.05$ ). Many of these ions were related directly (ornithine, carbamoyl-P, citrulline) or indirectly (aspartate, lysine, valine) to the urea cycle. Our results suggest that lower intake associated to low RFI led to a lower activity of the urea cycle in efficient growing heifers.
\end{abstract}

Keywords: feed efficiency, metabolomics, cattle, RFI

\section{Introduction}

Improving the feed efficiency in growing cattle is essential for enhancing the profitability and sustainability of the livestock sector (Ahola and Hill, 2012). Residual feed intake (RFI) is the preferred metric for genetic programs aiming at improving feed efficiency, but its measurement is labour, time consuming and sometimes not feasible in field conditions. Consequently, biomarkers need to be developed as tools to predict quickly and less costly the feed efficiency of a large number of animals. Recently, some studies have explored RFI biomarkers by non-targeted metabolomic approaches in fast-growing beef males (e.g. Karisa et al. 2014) but as far as we know, no studies have been yet conducted on heifers. However, this is particularly important, as improving feed efficiency in females will increase the overall cow/calf system efficiency by decreasing feeding costs. Thus, this study aimed at exploring biomarkers of RFI in growing heifers by a non-targeted metabolomics approach.

\section{Materials and methods}

One hundred and forty two Charolais beef heifers were individually phenotyped for body weight gain and intake during a test for RFI conducted across four experimental batches at the same INRA experimental farm. Heifers (age $=676 \pm 16$ days; body weight $=494 \pm 46 \mathrm{~kg}$ at the onset of the test) were adapted to diet during 4 weeks and then controlled over an 8 week test period with body weight recorded every 2 weeks. The experimental diet consisted in a TMR based on grass silage and concentrate at a ratio of 95:5 and supplying $5.4 \mathrm{MJ} \mathrm{NE} / \mathrm{kg}$ $\mathrm{DM}$ and $11.3 \% \mathrm{CP} / \mathrm{kg} \mathrm{DM}$. Blood was sampled from the coccygeal vessels on the last day of the RFI test. Blood was immediately centrifuged at $2500 \times \mathrm{g}$ for 10 minutes and the plasma harvested and stored at $-20^{\circ} \mathrm{C}$. Forty-eight extreme individuals were chosen based on RFI values (24 high RFI [1.59 $\pm 0.65 \mathrm{~kg}$ DMI/day] vs. 24 low RFI [-1.63 $\pm 0.68 \mathrm{~kg} \mathrm{DMI} /$ day]) to explore biomarkers of feed efficiency in blood. A non-targeted metabolomic approach (LCQtoF/MS, ESI +) was performed on plasma samples. Annotation was conducted using both 
the internal database of our laboratory and the Human-Metabolome Database (HMDB, http://www.hmdb.ca). The type of adduct, delta ( $\Delta \leq 10 \mathrm{ppm})$ and chemistry nature of the compounds was taken into account as criteria when annotation was made by HMDB. Metabolic pathways analyses were conducted using the MetScape app within the CytoScape software (Karnovsky et al., 2012).

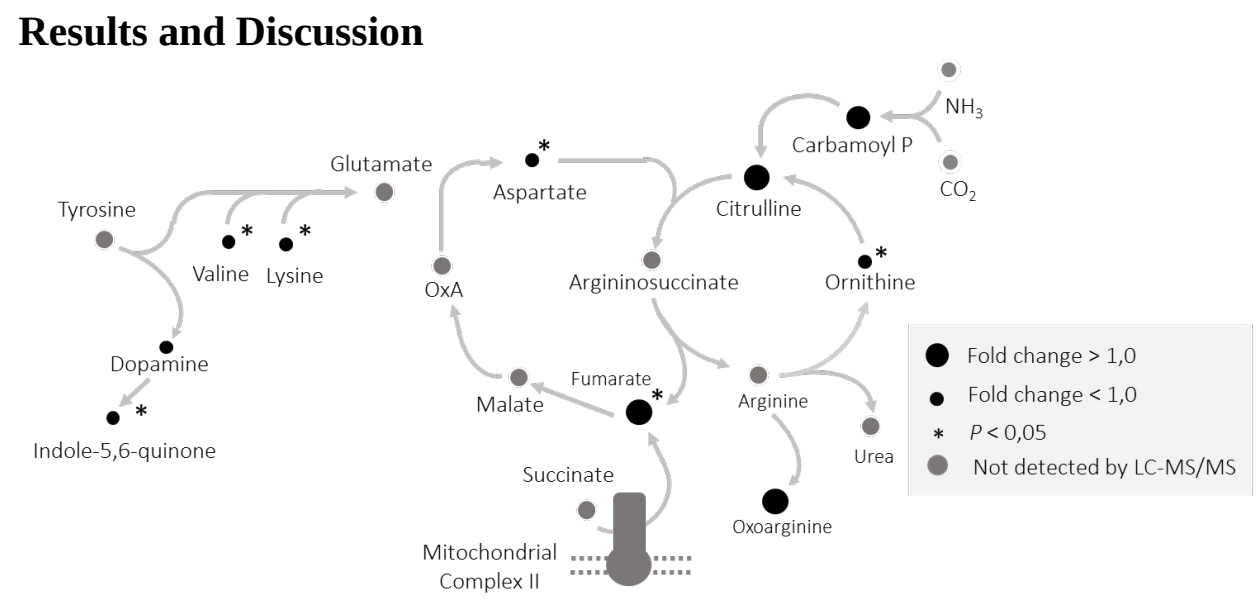

A total of 147 ions obtained by LC-MS/MS analysis changed across extreme RFI groups (74 with raw $P<0.05$ and 73 with $0.05<P<0.10$ ). Among them, 110 were annotated. We identified a common metabolic pathway including several of these annotated and discriminant ions, which 16 identified compounds corresponded to the urea cycle and amino acid (AA) metabolism (Figure 1). Our results suggest that urea cycle slowed down in efficient vs inefficient animals since two specific AA (Asp and Orn) involved in this cycle had lower $(P<$ 0.05 ) intensities and one necessary compound for the cycle (Carbomoyl-P) accumulated (fold change $=1.1, P=0.08$ ). In addition, the intensities of two essential AA (Val and Lys) which catabolism ends into the urea cycle decreased $(P<0.05)$ in efficient vs inefficient animals. Our results are in agreement with previous studies reporting a positive relationship between blood urea-N concentration (non-measured here) and RFI (Karisa et al., 2014). In addition, lower AA concentration in low vs. high RFI phenotypes have been previously reported (Karisa et al., 2014; Meale et al., 2017) and may reflect in part the lower AA absorption expected when dry matter, and thus protein, intake decreases.

Figure 1. Changes in the urea cycle pathway associated to residual feed intake in growing heifers revealed by metabolomics-based analyses. Black dots correspond to detected ions differing across RFI $(P<0.10)$. The most probable differences $(P<0.05)$ are depicted by a star while the dots size corresponds to the fold change between low vs high RFI groups.

\section{Aknowledgment}

The authors wish to thank APIS-GENE for their financial support of this project.

\section{Bibliography}

Ahola, J.K., R.A. Hill, 2012. Input factors affecting profitability: a changing paradigm and a challenging time. In: Hill, R.A. (ed.) Feed efficiency in the beef industry. Wiley-Blackwell, New Dehli, India, pp. 7-20.

Karisa, B.K., J. Thomson, Z. Wang, Z., C. Li, Y.R. Montanholi, S.P. Miller,S.S. Moore, G.Plastow, G. S., 2014. Plasma metabolites associated with residual feed intake and other productivity performance traits in beef cattle. Livestock Science 165, 200-211.

Meale, S.J., D.P. Morgavi, I. Cassar-Malek, D. Andueza, I. Ortigues-Marty, R. Robins, AM.Schiphorst, S.Laverroux, B. Graulet, H. Boudra, G. Cantalapiedra-Hijar, 2017. Exploration of Biological Markers of Feed Efficiency in Young Bulls. Journal of Agricultural and Food Chemistry 65, 9817-9827. 
Karnovsky A, T., T. Weymouth, V.G. Hull, G. Tarcea, C. Scardoni, M.A. Laudanna, K.A. Sartor, H.V. Stringer, H.V. Jagadish, C. Burant, B. Athey, G.S. Omenn, 2012. Metscape 2 bioinformatics tool for the analysis and visualization of metabolomics and gene expression data.

Bioinformatics 28(3), 373-380. 